\title{
A note on the Modified Log-Sobolev inequality.
}

\author{
Ioannis Papageorgiou*
}

\begin{abstract}
A criterion is presented for the Modified Logarithmic Sobolev inequality on metric measure spaces. The criterion based on U-bound inequalities introduced by Hebisch and Zegarlinski allows to show the inequality for measures that go beyond log-concavity.
\end{abstract}

Keywords: Modified Logarithmic Sobolev inequality.U bounds.

Mathematics Subject Classification (2000): 60E15 ·26D10

\section{Introduction.}

A lot of attention has been focused on inequalities that interpolate between the Log-Sobolev inequality

$$
\operatorname{Ent}_{\mu}\left(|f|^{r}\right) \leq C \mu|\nabla f|^{r} \quad\left(L S_{r}\right)
$$

(see [10], 2], 8]) for $r=2$ and the Spectral Gap inequality, that is

$$
\mu|f-\mu f|^{2} \leq C^{\prime} \mu|\nabla f|^{2} \quad(S G)
$$

where for the measure $\mu$ on $\mathbb{R}^{n}$, the entropy

$$
\operatorname{Ent}_{\mu}(f):=\mu\left(f \log \frac{f}{\mu f}\right)
$$

and $|\nabla f|$ is the Euclidean length of the gradient $\nabla f$ of the function $f: \mathbb{R}^{n} \rightarrow \mathbb{R}$. For a detailed account of these developments one can look on [11] and [6]. A first example of an inequality interpolating between the Log-Sobolev and the Spectral Gap was introduced by [7] and then studied by [16] and [5]. Modified Log-Sobolev inequalities have properties similar with the Log-Sobolev inequality, since they are

\footnotetext{
*Institut de Mathématiques de Toulouse, Université Paul Sabatier, 31062 Toulouse cedex 09, France. Email: ioannis.papageorgiou@math.univ-toulouse.fr
} 
both preserved under bounded perturbations and product measures (see [1], [8]), while they both imply concentration of measure properties (see [17], [11], [3], [6], [18]).

In this paper we are interested in the Modified Log-Sobolev inequality introduced by [11], (see also [12] and [6]).

Given a $q \in(2,+\infty)$, for $x \in \mathbb{R}$ we can define the modification $H_{q}$ of $|x|^{q}: \mathbb{R} \rightarrow$ $\mathbb{R}$ to be

$$
H_{q}(x)= \begin{cases}x^{2} & \text { if }|x| \leq 1 \\ |x|^{q} & \text { if } \quad|x| \geq 1\end{cases}
$$

The definition of the $\operatorname{MLS}\left(H_{q}\right)$ inequality follows.

The Modified Log-Sobolev Inequality.

We say that the measure $\mu$ satisfies the Modified Log-Sobolev Inequality if there exists a constant $C_{M L S}$ such that for any function $f \in C^{\infty}$ the following holds

$$
\mu|f|^{2} \log \frac{|f|^{2}}{\mu|f|^{2}} \leq C_{M L S} \int H_{q}\left(\frac{|\nabla f|}{f}\right) f^{2} d \mu \quad\left(M L S\left(H_{q}\right)\right)
$$

for some positive constant $C_{M L S}$.

Concerning measures that satisfy an $\operatorname{MLS}\left(H_{q}\right)$ inequality, in [11] it was shown that the $\left(\operatorname{MLS}\left(H_{q}\right)\right)$ inequality for the one dimensional measure on $\mathbb{R}$ is satisfied by the measure

$$
\mu_{p}=\frac{e^{-|x|^{p}}}{Z_{p}} d x
$$

for $p$ conjugate of $q$, i.e. $\frac{1}{p}+\frac{1}{q}=1$, and $Z_{p}$ the normalization constant. Furthermore, Barthe and Roberto in [6] showed that a measure $\frac{e^{-V(x)}}{\int e^{-V(x)} d x} d x$ such that

$$
\liminf _{|x| \rightarrow \infty} \operatorname{sign}(x) V^{\prime}(x)>0 \text { and } \lim _{|x| \rightarrow \infty} \frac{V^{\prime \prime}(x)}{V^{\prime}(x)^{2}}=0
$$

satisfies the $\left(\operatorname{MLS}\left(H_{q}\right)\right)$ inequality if and only if

$$
\lim _{|x| \rightarrow \infty} \frac{V(x)}{\left|V^{\prime}(x)\right|^{q}}<\infty
$$

As a consequence, one obtains that non log-concave measures like for instance

$$
V(x)=|x|^{p}+\alpha(x) \cos (x)
$$

such that $\alpha(x)=k|x|^{p-1-\delta}$ for $|x|>1, k$ a small constant, and $p \geq \frac{q}{q-1}$ and $\delta \in(0,1)$ satisfy the $\left(\operatorname{MLS}\left(H_{q}\right)\right)$ inequality. 
The purpose of this paper is to present examples where the $\left(\operatorname{MLS}\left(H_{q}\right)\right)$ inequality is satisfied for measures that go beyond the last mentioned example, as for instance measures defined with

$$
V(x)=d(x)^{p}+\alpha(x) \cos (x)
$$

such that $\alpha(x)=k d(x)^{p-1}$ for $|x|>1, k \in(0,1)$ some small constant and $p \geq \frac{q}{q-1}$, where $d(x)=d\left(x_{0}, x\right)$ denotes the distance of $x$ from a specific point $x_{0}$. In order to obtain such examples, at first a criterion is going to be presented based on the U-bound inequalities introduced by Hebisch and Zegarlinski in [14].

In the aforementioned paper, the U-bound inequalities

$$
\mu\left(f^{q} d^{s}\right) \leq C \mu|\nabla f|^{q}+D \mu|f|^{q}
$$

where used to prove Log-Sobolev $q$ inequalities for $q \in(1,2]$, the spectral Gap inequality, as well as F-Sobolev inequalities. In particular, for $\theta \in(1,2)$ the following U-bound inequality

$$
\mu_{\theta}\left(f^{2} d^{2(\theta-1)}\right) \leq C \mu_{\theta}|\nabla f|^{2}+D \mu_{\theta}|f|^{2}
$$

was used to prove the F-Sobolev inequality

$$
\int f^{2}\left|\log \frac{f^{2}}{\int f^{2} d \mu_{\theta}}\right|^{\varsigma} d \mu_{\theta} \leq C \mu_{\theta}|\nabla f|^{2}+D \mu_{\theta}|f|^{2}
$$

for $\varsigma \in[0,1]$ and $\mu_{\theta}=\frac{e^{-d^{\theta}(x)}}{\int e^{-d^{\theta}(x)} d x} d x$.

In this paper, we apply the methods developed in [14], in order to obtain similar results for the case of the Modified Logarithmic Sobolev inequality $\left(\operatorname{MLS}\left(H_{q}\right)\right.$ ). More detailed, as shown in Theorem 2.2 the following U-bound inequality for $q>2$

$$
\mu\left(f^{2} d^{q(p-1)}\right) \leq C \mu\left(|\nabla f|^{q}|f|^{2-q}\right)+D \mu|f|^{2}
$$

is sufficient for the measure $\mu$ to satisfy an $\left(\operatorname{MLS}\left(H_{q}\right)\right)$ inequality, for $p \geq q^{\prime}$, where $q^{\prime}$ is the conjugate of $q$. As a consequence, examples of non log-concave measures that satisfy a Modified Log-Sobolev inequality are presented in Corollary 2.3.

\section{A criterion on $\operatorname{MLS}\left(H_{q}\right)$ inequalities.}

We consider general $n$-dimensional non compact metric spaces. We will denote $d$ the distance and $\nabla$ the (sub)gradient for which we assume that $\frac{1}{\sigma}<|\nabla d| \leq 1$ for some $\sigma \in[1, \infty)$, and $\Delta d \leq K$ outside the unit ball $B=\{d(x)<1\}$ for some 
$K \in(0,+\infty)$. If $d \lambda$ is the $n$-dimensional Lesbegue measure we assume that it satisfies the Classical-Sobolev inequality (C-S)

$$
\left(\int|f|^{2+\epsilon} d \lambda\right)^{\frac{2}{2+\epsilon}} \leq \alpha \int|\nabla f|^{2} d \lambda+\beta \int|f|^{2} d \lambda
$$

for positive constants $\alpha, \beta$, as well as the local Poincaré inequality (see [19]), that is, there exists a constant $c_{R} \in(0, \infty)$ such that for every ball $B_{R}$,

$$
\frac{1}{\left|B_{R}\right|} \int_{B_{R}}\left|f-\frac{1}{\left|B_{R}\right|} \int_{B_{R}} f\right|^{2} d \lambda \leq c_{R} \frac{1}{B_{R}} \int_{B_{R}}|\nabla f|^{2} d \lambda
$$

Remark 2.1. The main assumptions for the n-dimensional non compact space with distance $d$ and (sub)gradient $\nabla$ are for instance satisfied in the case of the $\mathbb{R}^{n}, n \geq 1$ with $d$ being the Eucledian distance, as well as for the case of the Heisenberg group, with d being the Carnot-Carathéodory distance.

The local Poincaré inequality for the Lebesgue measure (2.1) is a standard result (see for instance [13], 14]). Concerning the Classical Sobolev inequality for $n \geq 3$ one can look at [9] and [20]. For the case of $n=1,2$ a stronger result holds. The $(C-S)$ in these two cases actually follows directly from the case $n=3$. To see this, for instance for $n=1$, if we consider a function $g: \mathbb{R} \rightarrow \mathbb{R}$ and then apply the $(C-S)$ inequality for $n=3$ for the function $f\left(x_{1}, x_{2}, x_{3}\right)=\prod_{i=1}^{3} g\left(x_{i}\right)$, we obtain

$$
\|g\|_{2+\epsilon}^{6} \leq \alpha\left(\int|\nabla g|^{2} d \lambda\right)\|g\|_{2}^{4}+\beta\|g\|_{2}^{6}
$$

If we assume $\|g\|_{2}^{2}=1$ we then have

$$
\|g\|_{2+\epsilon}^{2} \leq\left(\alpha \int|\nabla g|^{2} d \lambda+\beta\right)^{\frac{1}{3}}
$$

In the case where $\int|\nabla g|^{2} d \lambda \leq 1$, (2.2) becomes

$$
\|g\|_{2+\epsilon}^{2} \leq(\alpha+\beta)^{\frac{1}{3}}
$$

In the case where $\int|\nabla g|^{2} d \lambda>1$, from (2.2) we obtain

$$
\begin{aligned}
\|g\|_{2+\epsilon}^{2} & \leq\left(\alpha \int|\nabla g|^{2} d \lambda+\beta\right)^{\frac{1}{3}} \leq\left(\alpha \int|\nabla g|^{2} d \lambda\right)^{\frac{1}{3}}+\beta^{\frac{1}{3}} \\
& \leq \gamma \int|\nabla g|^{2} d \lambda+\beta^{\frac{1}{3}}
\end{aligned}
$$


for positive constants $\gamma$ and $\beta$. If we combine together inequalities (2.3) and 2.4) we have that for any $g: \mathbb{R} \rightarrow \mathbb{R}$ such that $\|g\|_{2}^{2}=1$ the following holds

$$
\|g\|_{2+\epsilon}^{2} \leq \gamma \int|\nabla g|^{2} d \lambda+(\alpha+\beta)^{\frac{1}{3}}
$$

for positive constants $\alpha, \beta, \gamma$. The result follows if we replace $g$ by $\frac{g}{\left(\int g^{2} d \lambda\right)^{\frac{1}{2}}}$.

Furthermore, for $d \lambda$ the Lesbegue measure, we define the probability measure

$$
d \mu_{p}=\frac{e^{-d^{p}}}{Z_{p}} d \lambda
$$

where $Z_{p}$ is the normalization constant. Since in this paper we are concerned with the subquadratic case we consider $1<p<2$. The main result is as follows.

Theorem 2.2. For any $q>2$, let $d \mu=\frac{e^{-W}}{Z} d \mu_{p}$ for $p \geq \frac{q}{q-1}$ be a probability measure defined with an a.e. differentiable potential $W$ satisfying

$$
|\nabla W| \leq \delta d^{p-1}+\gamma_{\delta}
$$

with some small constant $\delta \in(0,1)$ and $\gamma_{\delta} \in(0, \infty)$. Then the following Modified Log-Sobolev inequality $\left(M L S\left(H_{q}\right)\right)$ holds

$$
\mu|f|^{2} \log \frac{|f|^{2}}{\mu|f|^{2}} \leq c \int H_{q}\left(\frac{|\nabla f|}{f}\right) f^{2} d \mu
$$

for some positive constant $c$.

As a direct consequence of the last theorem, we can obtain examples of measures that are not log-concave and actually go beyond the examples provided in [6]. The corollary below presents such a family of measures.

Corollary 2.3. Let $d \mu=\frac{e^{-V}}{\int e^{-V} d \lambda} d \lambda$ be a probability measure defined with an a.e. differentiable potential $V$ satisfying

$$
V(x)=d(x)^{p}+\alpha(x) \cos (x)
$$

such that $\alpha(x)=k d(x)^{p-1}$ for $|x|>1$, where $p \geq \frac{q}{q-1}$ and $k \in(0,1)$ a small constant. Then the following Modified Log-Sobolev inequality holds

$$
\mu|f|^{2} \log \frac{|f|^{2}}{\mu|f|^{2}} \leq c \int H_{q}\left(\frac{|\nabla f|}{f}\right) f^{2} d \mu
$$


As explained in the introduction, the $U$-bound inequalities introduced in [14] will play a crucial role in proving the MLS inequality. The proposition bellow provides a link between subquadratic measures and $U$-bound inequalities.

Proposition 2.4. Let $d \mu=\frac{e^{-W}}{Z} d \mu_{p}$ be a probability measure defined with a differentiable potential $W$ satisfying

$$
|\nabla W| \leq \delta d^{p-1}+\gamma_{\delta}
$$

with some small constant $\delta \in(0,1)$ and $\gamma_{\delta} \in(0, \infty)$. Then there exist constants $C^{\prime}, D^{\prime} \in(0,+\infty)$ such that the following bound holds

$$
\int|f|^{2} d^{q(p-1)} d \mu \leq C \int H_{q}\left(\frac{|\nabla f|}{f}\right) f^{2} d \mu+D \int|f|^{2} d \mu
$$

Proof. The starting point of the proof is the following U-bound inequality from [14.

Theorem 2.5. ([14]) Assume that $\frac{1}{\sigma}<|\nabla d| \leq 1$ for some $\sigma \in[1, \infty)$, and $\Delta d \leq K$ outside the unit ball $B=\{d(x)<1\}$ for some $K \in(0,+\infty)$. Let $d \mu=\frac{e^{-W}}{Z} d \mu_{p}$ be a probability measure defined with a differentiable potential $W$ satisfying

$$
|\nabla W| \leq \delta d^{p-1}+\gamma_{\delta}
$$

with some small constant $\delta \in(0,1)$ and $\gamma_{\delta} \in(0, \infty)$. Then there exist constants $C^{\prime}, D^{\prime} \in(0,+\infty)$ such that the following bound holds

$$
\int|f| d^{p-1} d \mu \leq C^{\prime} \int|\nabla f| d \mu+D^{\prime} \int|f| d \mu
$$

We will use the last theorem in order to obtain a $U$-bound inequality with a tighter left hand side. Let $d_{1}(x)=\max (1, d(x))$. From Theorem 2.5, by enlarging the constant $D^{\prime}$ we may assume that

$$
\int|f| d_{1}^{p-1} d \mu \leq C^{\prime} \int|\nabla f| d \mu+D^{\prime} \int|f| d \mu
$$

If we choose $h=|f|^{2} d_{1}^{(q-1)(p-1)}$ we have

$$
\int|f|^{2} d^{q(p-1)} d \mu \leq \int|f|^{2} d_{1}^{q(p-1)} d \mu
$$

Furthermore, from the inequality (2.6) we can obtain the following bound

$$
\int|f|^{2} d_{1}^{q(p-1)} d \mu=\int|h| d_{1}^{p-1} d \mu \leq C^{\prime} \int|\nabla h| d \mu+D^{\prime} \int|h| d \mu
$$


For $R>1$ we have

$$
\begin{aligned}
\int h d \mu & =\int|f|^{2} d_{1}^{(p-1)(q-1)} d \mu \\
& =\int_{B_{R}}|f|^{2} d_{1}^{(p-1)(q-1)} d \mu+\int_{B_{R}^{c}}|f|^{2} d_{1}^{(p-1)(q-1)} d \mu
\end{aligned}
$$

where $B_{R}$ denotes a ball of radius $R$, i.e. $B_{R}=\{d(x)<R\}$. For the first term on the right hand side of (2.9) we have

$$
\int_{B_{R}}|f|^{2} d_{1}^{(p-1)(q-1)} d \mu \leq R^{(p-1)(q-1)} \int|f|^{2} d \mu
$$

While for the second term on the right hand side of (2.9) we compute

$$
\int_{B_{R}^{c}}|f|^{2} d_{1}^{(p-1)(q-1)} d \mu \leq \frac{1}{R^{p-1}} \int|f|^{2} d_{1}^{q(p-1)} d \mu
$$

From (2.9)-(2.11) we finally obtain

$$
\int h d \mu=R^{(p-1)(q-1)} \int|f|^{2} d \mu+\frac{1}{R^{p-1}} \int|f|^{2} d_{1}^{q(p-1)} d \mu
$$

for a constant $R>1$. Furthermore, we have

$$
|\nabla h|=2|\nabla f||f| d_{1}^{(p-1)(q-1)}+(q-1)(p-1)\left|\nabla d_{1}\right||f|^{2} d_{1}^{(p-1)(q-1)-1}
$$

We can compute

$$
\begin{aligned}
\int 2|\nabla f||f| & d_{1}^{(p-1)(q-1)} d \mu \\
& =\int 2|\nabla f||f|^{\frac{2}{q}-1}|f|^{2 \frac{(q-1)}{q}} d_{1}^{(p-1)(q-1)} d \mu \\
& \leq 2\left(\int|\nabla f|^{q}|f|^{2-q} d \mu\right)^{\frac{1}{q}}\left(\int|f|^{2} d_{1}^{\frac{q(p-1)(q-1)}{q-1}} d \mu\right)^{\frac{q-1}{q}} \\
& \leq \alpha^{2} \int|\nabla f|^{q}|f|^{2-q} d \mu+\frac{1}{\alpha^{2}} \int|f|^{2} d_{1}^{q(p-1)} d \mu
\end{aligned}
$$

for a constant $\alpha>0$. We also have

$$
\begin{aligned}
\int\left|\nabla d_{1}\right||f|^{2} d_{1}^{(p-1)(q-1)-1} d \mu & \leq \int f^{2} d_{1}^{(p-1)(q-1)} d \mu \\
& \leq R^{(p-1)(q-1)} \int f^{2} d \mu+\frac{1}{R^{p-1}} \int|f|^{2} d_{1}^{q(p-1)} d \mu
\end{aligned}
$$


where above we used the bound from (2.12). From (2.13), (2.14) and (2.15) we obtain

$$
\begin{aligned}
\int|\nabla h| d \mu \leq & \alpha^{2} \int|\nabla f|^{q}|f|^{2-q} d \mu+(q-1)(p-1) R^{(p-1)(q-1)} \int|f|^{2} d \mu \\
& +\left(\frac{1}{\alpha^{2}}+\frac{(q-1)(p-1)}{R^{p-1}}\right) \int|f|^{2} d_{1}^{q(p-1)} d \mu
\end{aligned}
$$

If we plug (2.16) and (2.12) in (2.8) we finally obtain

$$
\begin{aligned}
\int|f|^{2} d_{1}^{q(p-1)} d \mu \leq & C^{\prime} \alpha^{2} \int|\nabla f|^{q}|f|^{2-q} d \mu \\
& +\left(D^{\prime}+C^{\prime}(q-1)(p-1)\right) R^{(p-1)(q-1)} \int|f|^{2} d \mu \\
& +\left(\frac{C^{\prime}}{\alpha^{2}}+\frac{D^{\prime}+C^{\prime}(q-1)(p-1)}{R^{p-1}}\right) \int|f|^{2} d_{1}^{q(p-1)} d \mu
\end{aligned}
$$

If we choose $\alpha$ and $R$ large enough so that $\frac{C^{\prime}}{\alpha^{2}}+\frac{D^{\prime}+C^{\prime}(q-1)(p-1)}{R^{p-1}}<1$ we obtain

$$
\int|f|^{2} d_{1}^{q(p-1)} d \mu \leq C \int|\nabla f|^{q}|f|^{2-q} d \mu+\breve{D} \int f^{2} d \mu
$$

for constants

$$
C=\frac{C^{\prime} \alpha}{1-\left(\frac{C^{\prime}}{\alpha^{2}}+\frac{D^{\prime}+C^{\prime}(q-1)(p-1)}{R^{p-1}}\right)}
$$

and

$$
\breve{D}=\frac{\left(D^{\prime}+C^{\prime}(q-1)(p-1)\right) R^{(p-1)(q-1)}}{1-\left(\frac{C^{\prime}}{\alpha^{2}}+\frac{D^{\prime}+C^{\prime}(q-1)(p-1)}{R^{p-1}}\right)}
$$

The proof of the proposition follows from (2.7) and (2.18) for constant $D=C+\breve{D}$, since $|\nabla f|^{q}|f|^{2-q} \leq f^{2}$ when $|\nabla f| \leq|f|$.

If we compare the U-bound inequality (2.5) of Proposition 2.4 with the U-bound inequality (1.1) used in [14] to show the F-Sobolev inequality (1.2), one notices that the left hand side of (2.5) is stronger, while the right hand side is relaxed from the full gradient to the weaker modification related with the Modified Logarithmic Sobolev inequality $\left(\operatorname{MLS}\left(H_{q}\right)\right)$. In the next proposition we present the link between the $U$-bound inequality of the last proposition and the Defective Modified LogSobolev inequality. 
Proposition 2.6. Suppose that the measure

$$
d \mu=\frac{e^{-U} d \lambda}{\int e^{-U} d \lambda}
$$

where $d \lambda$ the $n$-dimensional Lebesgue measure and $U \geq 0$, satisfies the following U-bound inequality

$$
\mu|f|^{2}\left(|\nabla U|^{2}+U\right) \leq \hat{C} \int H_{q}\left(\frac{|\nabla f|}{f}\right) f^{2} d \mu+\hat{D} \mu|f|^{2}
$$

for some positive constants $\hat{C}$ and $\hat{D}$ both independent of $f$. Then the following Defective Modified Log-Sobolev inequality holds

$$
\mu|f|^{2} \log \frac{|f|^{2}}{\mu|f|^{2}} \leq C \int H_{q}\left(\frac{|\nabla f|}{f}\right) f^{2} d \mu+D \mu f^{2}
$$

Proof. We follow closely the work in [14] for the Log-Sobolev $q$ inequality (see also [15]). Without loss of generality we can assume that $f \geq 0$ and we set $\rho=\frac{e^{-U}}{\int e^{-U} d \lambda}$ and $g=f \rho^{\frac{1}{2}}$ We also assume that

$$
\int g^{2} d \lambda=\mu f^{2}=1
$$

Then we can write

$$
\int\left(g^{2} \log g^{2}\right) d \lambda=\frac{2}{\epsilon} \int g^{2}\left(\log 2^{\epsilon}\right) d \lambda \leq \frac{2+\epsilon}{2} \frac{2}{\epsilon} \log \left(\int g^{2+\epsilon} d \lambda\right)^{\frac{2}{2+\epsilon}}
$$

where above we used the Jensen's inequality. If we use now the Classical-Sobolev inequality (C-S) for the Lebesgue measure $d \lambda$

$$
\left(\int|f|^{2+\epsilon} d \lambda\right)^{\frac{2}{2+\epsilon}} \leq \alpha \int|\nabla f|^{2} d \lambda+\beta \int|f|^{2} d \lambda
$$

for positive constants $\alpha, \beta$, we will get

$$
\begin{aligned}
\int\left(g^{2} \log g^{2}\right) d \lambda & \leq \frac{2+\epsilon}{\epsilon} \log \left(\alpha \int|\nabla g|^{2} d \lambda+\beta \int|g|^{2} d \lambda\right) \\
& \leq \frac{(2+\epsilon) \alpha}{\epsilon} \int|\nabla g|^{2} d \lambda+\frac{(2+\epsilon) \beta}{\epsilon} \int|g|^{2} d \lambda
\end{aligned}
$$


where in the last inequality we used that $\log x \leq x$ for $x>0$. For the first term on the right hand side of (2.20) we have

$$
\begin{aligned}
\int|\nabla g|^{2} d \lambda & =\int\left|\nabla\left(f \rho^{\frac{1}{2}}\right)\right|^{2} d \lambda \\
& \leq 2^{q-1} \mu|\nabla f|^{2}+2 \int\left|f \nabla\left(\rho^{\frac{1}{2}}\right)\right|^{2} d \lambda
\end{aligned}
$$

We have

$$
\begin{aligned}
\int\left|f \nabla\left(\rho^{\frac{1}{2}}\right)\right|^{2} d \lambda & =\int\left|\rho^{\frac{1}{2}} \rho^{\frac{-1}{2}} f \nabla\left(\rho^{\frac{1}{2}}\right)\right|^{2} d \lambda=\mu f^{2}\left|\rho^{\frac{-1}{2}} \nabla\left(\rho^{\frac{1}{2}}\right)\right|^{2} \\
& =\frac{1}{4} \mu f^{2}|\nabla U|^{2}
\end{aligned}
$$

If we plug the last equality in (2.21), we obtain

$$
\int|\nabla g|^{2} d \lambda \leq 2 \mu|\nabla f|^{2}+\frac{1}{2} \mu f^{2}|\nabla U|^{2}
$$

If we combine inequalities (2.20) and (2.22), we get

$$
\begin{aligned}
\int\left(g^{2} \log g^{2}\right) d \lambda \leq & \frac{2(2+\epsilon) \alpha}{\epsilon} \mu|\nabla f|^{2}+\frac{(2+\epsilon) \beta}{\epsilon} \mu f^{2} \\
& +\frac{2(2+\epsilon) \alpha}{\epsilon 4} \mu f^{2}|\nabla U|^{2}
\end{aligned}
$$

For the left hand side of (2.23), since $U \geq 0$, we have

$$
\begin{aligned}
\int\left(g^{2} \log g^{2}\right) d \lambda & =\int\left(\frac{e^{-U}}{\int e^{-U} d \lambda} f^{2} \log \frac{e^{-U}}{\int e^{-U} d X} f^{2}\right) d \lambda \\
& =\mu\left(f^{2} \log f^{2}\right)+\mu\left(f^{2} \log \frac{e^{-U}}{\int e^{-U} d \lambda}\right) \\
& =\mu\left(f^{2} \log f^{2}\right)-\mu\left(f^{2} U\right)-\mu\left(f^{2} \log \int e^{-U} d \lambda\right) \\
& \geq \mu\left(f^{2} \log f^{2}\right)-\mu\left(f^{2} U\right)
\end{aligned}
$$

If we combine (2.23) and (2.24), we obtain

$$
\mu\left(f^{2} \log f^{2}\right) \leq \hat{\alpha} \mu|\nabla f|^{2}+\hat{\gamma} \mu f^{2}+\hat{\beta} \mu f^{2}\left(|\nabla U|^{2}+U\right)
$$

where $\hat{\alpha}=\frac{2(2+\epsilon) \alpha}{\epsilon}, \hat{\beta}=\max \left\{\frac{2(2+\epsilon) \alpha}{\epsilon 4}, 1\right\}$ and $\hat{\gamma}=\frac{(2+\epsilon) \beta}{\epsilon}$. If we use the U-bound (2.19), the inequality (2.25) gives

$$
\mu\left(f^{2} \log f^{2}\right) \leq(\hat{\alpha}+\hat{\beta} \hat{C}) \int H_{q}\left(\frac{|\nabla f|}{f}\right) f^{2} d \mu+\hat{\beta} \hat{D} \mu f^{2}+\hat{\gamma} \mu f^{2}
$$

If we replace $f$ with $\frac{f}{\mu f}$ which has mean equal to one we obtain the result. 
We can now present the proof of Theorem 2.2 .

Proof of Theorem 2.2. Since $p \geq \frac{q}{q-1}$ and $|\nabla W| \leq \delta d^{p-1}+\gamma_{\delta}$ for a small constant $\delta \in(0,1)$ and $\gamma_{\delta} \in(0, \infty)$ we derive that for $U=d^{p}+W$ there exists a positive constant $\tilde{C}$ such that

$$
\int f^{2}\left(|\nabla U|^{2}+U\right) d \mu \leq \tilde{C} \int f^{2} d^{q(p-1)} d \mu+\tilde{C} \int f^{2} d \mu
$$

since $q>2$ and $p \geq \frac{q}{q-1}$. If we apply the U-bound inequality of Proposition 2.4 to bound the first term on the right hand side of the last inequality, we then get

$$
\int f^{2}\left(|\nabla U|^{2}+U\right) d \mu \leq \tilde{C} C \int H_{q}\left(\frac{|\nabla f|}{f}\right) f^{2} d \mu+\tilde{C}(D+1) \int|f|^{2} d \mu
$$

Since we have obtained a U-bound inequality like the one required in hypothesis (2.19) we can apply Proposition 2.6. This will lead to the Defective Modified Log-Sobolev inequality

$$
\int f^{2} \log \frac{f^{2}}{\int f^{2} d \mu} d \mu \leq C \int f^{2} H_{q}\left(\frac{|\nabla f|}{f}\right) d \mu+D \int f^{2} d \mu
$$

for positive constants $C$ and $D$. In order to finish the proof of the theorem it remains to pass from the Defective Modified Log-Sobolev inequality (2.26) to the Modified Log-Sobolev inequality $\left(\operatorname{MLS}\left(H_{q}\right)\right)$. To do this we will first need the Poincaré inequality for the measure $d \mu$. This is provided from the following theorem, whose proof can be found in [14].

Theorem 2.7. ([14]) Suppose $1 \leq q<\infty$ and a measure $\lambda$ satisfies the $q$-Poincaré inequality for every ball $B_{R}$, that is there exists a constant $c_{R} \in(0, \infty)$ such that

$$
\frac{1}{\left|B_{R}\right|} \int_{B_{R}}\left|f-\frac{1}{\left|B_{R}\right|} \int_{B_{R}} f\right|^{q} d \lambda \leq c_{R} \frac{1}{B_{R}} \int_{B_{R}}|\nabla f|^{q} d \lambda
$$

Let $\mu$ be a probability measure which is absolutely continuous with respect to the measure $\lambda$ and such that

$$
\int|f|^{q} \eta d \mu \leq C \int|\nabla f|^{q}+D \int|f|^{q} d \mu
$$

with some nonnegative function $\eta$ and some constants $C, D \in(0, \infty)$ independent of a function $f$. If for any $L \in(0, \infty)$ there is a constant $A_{L}$ such that

$$
\frac{1}{A_{L}} \leq \frac{d \mu}{d \lambda} \leq A_{L}
$$


on the set $\{\eta<L\}$ and, for some $R \in(0, \infty)$ (depending on $L)$, we have $\{\eta<$ $L\} \subset B_{R}$, then $\mu$ satisfies the q-Poincaré inequality

$$
\mu|f-\mu f|^{q} \leq c^{\prime} \mu|\nabla f|^{q}
$$

For $\lambda$ being the Lebesgue measure, (2.27) is true as initially assumed in (2.1). Furthermore, (2.28) was shown in Proposition 2.4 for $\eta=d^{q(p-1)}$. Thus, if we apply the above theorem for $q=2$ we obtain that the measure $\mu$ also satisfies the following Spectral Gap inequality

$$
\mu|f-\mu f|^{2} \leq c^{\prime} \mu|\nabla f|^{2}
$$

for some positive constant $c^{\prime}$. If we combine the Defective Modified Logarithmic Sobolev inequality (2.26), together with the Spectral Gap inequality (2.29) the theorem follows according to the following theorem (see [4])

Theorem 2.8. ([4]) Let $H$ be an even function on $\mathbb{R}$, which is increasing on $\mathbb{R}^{+}$ and satisfies $H(0)=0$ and $H(x) \geq c x^{2}$. Assume that there exists $q \geq 2$ such that $x \rightarrow \frac{H(x)}{x^{q}}$ is non-increasing on $(0,+\infty)$.

Assume that a probability measure $\mu$ satisfies a Defective Modified Log-Sobolev inequality:

$$
\int f^{2} \log \frac{f^{2}}{\int f^{2} d \mu} d \mu \leq \int f^{2} H\left(\frac{|\nabla f|}{f}\right) d \mu+D \int f^{2} d \mu
$$

If $\mu$ also satisfies a Poincaré inequality, then there exists a constant $C$ such that for every $f$,

$$
\int f^{2} \log \frac{f^{2}}{\int f^{2} d \mu} d \mu \leq C \int f^{2} H\left(\frac{(|\nabla f|}{f}\right) d \mu
$$

\section{Conclusion.}

In this paper a criterion on the Modified Log-Sobolev inequality was presented with the use of U-bounds. In particular, in Proposition 2.6 we saw that the following U-bound inequality

$$
\mu|f|^{2}\left(|\nabla U|^{2}+U\right) \leq \hat{C} \mu\left(H_{q}\left(\frac{|\nabla f|}{f}\right) f^{2}\right)+\hat{D} \mu|f|^{2}
$$

together with the Spectral Gap are sufficient for the measure $d \mu=\frac{e^{-U} d \lambda}{\int e^{-U} d \lambda} d \lambda$ to satisfy a $\operatorname{MLS}\left(H_{q}\right)$ inequality. Concerning the converse problem, if we follow the work in 14 for the similar problem concerning the stronger Log-Sobolev $q$ inequalities we can show the following result. 
Theorem 3.1. Suppose that the measure $d \mu=\frac{e^{-U} d \lambda}{\int e^{-U} d \lambda} d \lambda$ satisfies an $M L S\left(H_{q}\right)$ inequality and that $U$ is such that

$$
|\nabla U|^{q} \leq a U+b
$$

for positive constants $a$ and $b$. Then the following U-bound is true

$$
\int|f|^{2} U d \mu \leq C \int H_{q}\left(\frac{|\nabla f|}{f}\right) f^{2} d \mu+D \int|f|^{2} \mu
$$

This demonstrates that as in the case of Log-Sobolev $q$ and F-Sobolev inequalities, the Modified $\log -\operatorname{Sobolev} \operatorname{MLS}\left(H_{q}\right)$ inequalities are also equivalent to U-bound inequalities.

\section{References}

[1] C. Ané, S. Blachère, D. Chafaï, P. Fougères, I. Gentil, F. Malrieu, C. Roberto, G. Scheffer, Sur les inégalités de Sobolev logarithmiques. Panoramas et Synthèses. Soc. Math, 10, France, Paris (2000)

[2] D. Bakry, L'hypercontractivitè et son utilisation en théorie des semigroups. Séminaire de Probabilités XIX, Lecture Notes in Math., 1581, Springer, New York, 1-144 (1994)

[3] F. Barthe, P. Cattiaux and C. Roberto, Interpolating inequalities between exponential and Gaussian, Orlicz hypercontractivity and isoperimetry. Rev. Mat. Iberoamericana, 22, 993-1067 (2006)

[4] F. Barthe and A. Kolesnikov, Mass Transport and Variants of the Logarithmic Sobolev Inequality. J. Geom. Anal., 18, 921-979 (2008)

[5] F. Barth and C. Roberto, Sobolev Inequalities for probability measures on the real line. Studia Math., 159, 481-497 (2003)

[6] F. Barth and C. Roberto, Modified Logarithmic Sobolev Inequality on $\mathbb{R}$. Potential Anal., 29, 167-193 (2008)

[7] W. Beckner, A generalised Poincaré inequality for Gaussian measures. Proc. Amer. Math. Soc., 105(2), 397 - 400 (1989)

[8] S.G. Bobkov and B. Zegarlinski, Entropy Bounds and Isoperimetry. Memoirs of the American Mathematical Society, 176, 1 - 69 (2005) 
[9] E.B. Davies, Heat Kernels and Spectral Theory. Cambridge Tracts in Mathematics, 92, CUP (1990)

[10] L. Gross, Logarithmic Sobolev inequalities. Am. J. Math., 97, 1061-1083 (1976)

[11] I. Gentil, A. Guillin and L. Miclo, Modified logarithmic Sobolev inequalities and transportation inequalities. Probab.Theor. Relat. Fields 133, 409-436 (2005).

[12] I. Gentil, A. Guillin and L. Miclo, Modified logarithmic Sobolev inequalities in null curvature. Rev. Mat. Iberoamericana, 23(1), 235-258 (2007).

[13] E. Hebey, Sobolev Spaces on Riemannian Manifolds. Lecture Notes in Math. 1635, Springer (1996)

[14] W. Hebisch and B. Zegarlinski, Coercive inequalities on metric measure spaces. J. Func. Anal., 258, 814-851 (2010).

[15] J. Inglis and I. Papageorgiou, Logarithmic Sobolev Inequalities for Infinite Dimensional Hörmander Type Generators on the Heisenberg Group. Potential Anal., 31, 79-102 (2009)

[16] R. Latala and K. Oleszkiewicz, Between Sobolev and Poincaré. In Geometric aspects of functional analysis, Lecture Notes in Math. 1745, 147 - 168, Springer, Berlin (2000)

[17] M. Ledoux, Concentration of measure and logarithmic Sobolev inequalities. Séminaire de Probabilités, XXXIII, Lecture Notes in Math. 1709, Springer, 120216. (1999)

[18] I. Papageorgiou, Concentration inequalities for Gibbs measures. Infin. Dimens. Anal. Quantum Probab. Relat. Top. (to appear)

[19] L. Saloff-Coste, Aspects of Sobolev Type Inequalities. London Mathematical Society Lecture Notes 289, Cambridge University Press, Cambridge (2002).

[20] N.T. Varopoulos, L. Saloff-Coste and T. Coulhon, Analysis and geometry on groups. Cambridge Tracks in Mathematics, 100, Cambridge University Press, Cambridge (1992). 